be under estimated by their supervisor while they are supported by their colleagues. In addition, most of them felt unsatisfied with their salary and were not confident in their career evolution.

Conclusion The discrepancy raised by our study between the real workload and the perceived well-being seems to be conditioned by organizational dysfunction and dissatisfaction with conditions of work performance. New organizational possibilities with brainstorming approaches of the nurses' work environment are recommended and are being built through interdisciplinary research-action studies.

\section{P-171 AIR RECIRCULATION IN VENTILATION SYSTEM AND ITS IMPACT ON TRICHLORAMINE EXPOSURES IN A SWIMMING POOL HALL: A NUMERICAL INVESTIGATION}

${ }^{1}$ Hélène Proulx, Maximilien Debia, Stéphane Hallé. 'École de technologie supérieure, Canada

\subsection{6/OEM-2021-EPI.218}

Introduction Trichloramine $(\mathrm{NCl} 3)$ is an irritant gas commonly found in the air of indoor swimming pool, causing health problems to swimmers and workers who are often exposed to this contaminant. ASHRAE recommends a supply air delivery rate of 4 to 8 air changes per hour to remove trichloramine in aquatic centers. However, the fraction of recirculated air can have a significant impact on the exposure level.

Objective The main objective of this study is to investigate the impact of air recirculation by mechanical ventilation systems on $\mathrm{NCl} 3$ exposures for five user groups: i) swimmers in the basin, ii) people sitting or iii) standing on the deck, iv) lifeguards in surveillance chairs and v) spectators in mezzanine area.

Methods Exposure to $\mathrm{NCl} 3$ levels in a $9300 \mathrm{~m} 3$ swimming pool hall located in Montreal (Canada) is evaluated with a computational fluid dynamic software (Fire Dynamic Simulator). Simulations are performed for five recirculation ratios starting from the reference case at $77 \%$ down to $0 \%(100 \%$ fresh air). Emission rate of $\mathrm{NCl} 3$ is based on equations found in literature for a fully occupied pool. The numerical model was experimentally validated with tracer gas injection in situ.

Results The tracer gas simulation results show good agreement with the experimental results. Compared to the reference case (77\% recirculation condition), a 100\% fresh air ventilation strategy decreases the $\mathrm{NCl} 3$ levels of the five groups from: i) $19 \%$, ii) $31 \%$, iii) $62 \%$, iv) $61 \%$ and v) $77 \%$, respectively.

Conclusion Reducing the recirculation rate reduces the $\mathrm{NCl} 3$ concentrations. However, recirculation of air has less impact on the concentrations in the breathing zones located near the water surface compared to the other zones. The best air quality improvement with the $100 \%$ fresh air strategy is for spectators. Other ventilation strategies have to be investigated to improve air quality in the lower breathing zones as well.

\section{P-175 UNDERSTANDING THE ASSOCIATION BETWEEN TREATMENT MODALITY VERSUS EMPLOYMENT STATUS AMONG PATIENTS WITH ORGAN FAILURE}

${ }^{1}$ Anil Adisesh, Vijay Kumar Chattu, Sumaya Dano, Istvan Musci, Nathaniel Edwards. ${ }^{1}$ University of Toronto, Canada

10.1136/OEM-2021-EPI.219
Objectives In advanced organ failure patients: (1) To study the association between treatment modality versus employment status, (2) To analyze socio-demographic profiles and deprivation status, and (3) To explore Return to Work (RTW) by analysis of self-reported employment status.

Methods This ongoing hospital based cross-sectional study used a convenience sample: 1070 patients with advanced organ failure requiring solid organ transplant (SOT) or dialysis pooled from the Comprehensive Psychosocial Research Data System at UHN, Toronto. Patient reported outcome measures, clinical and socio-demographic variables were collected from stable outpatients on iPads using electronic data capture (DAta Driven Outcomes System-DADOS, Techna Institute, UHN, Toronto). The Ontario Marginalization Index characterized material deprivation. Data was analyzed using STATA v16, logistic regression used multiple imputation by chained equations.

Results Of 1070 SOT recipients, 646 (60\%) were males with $424(40 \%)$ females. Almost half (49\%) were of White ethnicity, Black (23\%) followed by South Asians and East Asians. 63\% (646) had >12 years education, the kidneypancreas sub-group were highest $88 \%$ (30) then kidney, liver and dialysis with $65 \%, 62 \%$ and $59 \%$, respectively. $51 \%$ of dialysis patients had a yearly income of $\$ 70,000$. These results reflect the higher employment status of kidney (60\%), liver (55\%) and kidney-pancreas (51\%) transplants compared to dialysis patients (28\%). 59\% of dialysis patients had high deprivation. The association between treatment modality vs employment status and employment status vs deprivation remained statistically significant after adjusting for age, sex, ethnicity and comorbidities in logistic regression models.

Conclusion SOT was associated with significantly higher odds of employment and lower material deprivation. Dialysis, transplant and occupational health professionals should support SOT and dialysis patients overcoming barriers to maintain and RTW. RTW post-SOT is complex and likely associated with personal, professional, societal and medical factors.

\section{P-177 TEMPORARY AND PERMANENT AUDITORY EFFECTS ASSOCIATED WITH OCCUPATIONAL CO-EXPOSURE TO LOW LEVELS OF SOLVENTS AND NOISE}

${ }^{1}$ Adriana Lacerda, Vanessa Bohn, Maria Renata José, Simone Mariotti Roggia, Fernanda Zuck, Benoît Pouyatos, Thomas Venet, Edward Krieg, Thais C Morata. ${ }^{1}$ Universidade Tuiuti do Parana, Brazil

\subsection{6/OEM-2021-EPI.220}

Introduction The effect of combined exposures to noise and solvents on hearing has been studied for decades, but the characterization of the risk is incomplete.

Objective To assess the temporary and permanent auditory effects associated with occupational co-exposures to low levels of solvents and noise.

Method Cross-sectional study with 25 printing industry workers (mean age 36.2 years) simultaneously exposed to low levels of solvents and noise $(<8 \quad 0$ dBA TWA). The control group composed of 29 industry workers (mean age 36.7 years) without exposure to noise and/or solvents. Participants answered a questionnaire and underwent pure-tone audiometry (PTA), acoustic immittance tests, auditory brainstem response 
(ABR), dichotic digit test (DD). We examined auditory fatigue through the EchoScan test.

Results Exposed workers had worse thresholds bilaterally at 4 $\mathrm{kHz}(\mathrm{p}=0.0253$ in right ear - RE; $\mathrm{p}=0.0436$ in left ear LE). Ipsilateral acoustic reflex levels were different between groups at the frequencies of $500 \mathrm{~Hz}(p=0.02$ in RE; $p=0.04$ in LE) and $1 \mathrm{kHz}(\mathrm{p}=0.04$ in LE). The only differences detected by the $\mathrm{ABR}$ was for the interpeak interval III-V $(p=0.03$ in RE; $p=0.02$ in LE). DD means (exposed $=89.5$ \pm 13.33 ; control $=96.40 \pm 4.46$ ), were only different in the LE $(p=0.05)$. Echoscan detected a difference $(p=0.0317)$ between the means of the exposed $(4.58 \pm 6.8)$ and control $(0 \pm 4.62)$ groups, indicating possible hearing fatigue in the exposed group.

Conclusion Each of the hearing tests indicated differences between the groups, suggesting that occupational co-exposure to low noise and low concentrations of solvents can be harmful to hearing functions. The temporary effect of the exposure was detected by the EchoScan and the acoustic reflex of the stapedius muscle. The permanent effects were identified mainly at the level of the high brainstem and in the auditory ability of binaural integration. The EchoScan, can facilitate the early identification of auditory effects, while these are still temporary.

\section{P-178 EXPOSURE-RESPONSE RELATIONSHIPS FOR WOOD DUST EXPOSURE AND WORK-RELATED ASTHMA IN MOZAMBIQUAN WOOD PROCESSING WORKERS}

${ }^{1}$ Paulino Chamba, Roslynn Baatjies, Mohamed Jeebhay. 'Centre of Medical Examinations, Mozambique

\subsection{6/OEM-2021-EPI.221}

Introduction While exposure-response relationships for workrelated asthma have been studied in wood processing workers, few studies have used multiple exposure indices to evaluate various clinical endpoints.

Objectives To investigate exposure-response relationships for work-related respiratory symptoms, airway obstruction, bronchial hyperresponsiveness, and exhaled nitric oxide (FeNO) among Mozambiquan wood processing workers.

Methods A cross-sectional study of 450 workers using inhalable wood dust particulate concentrations assigned to workers based on personal environmental exposure samples $(n=124)$ collected for different jobs. Exposure metrics of current and cumulative dust exposure were modeled using multivariate linear and logistic regression for the clinical endpoints, adjusted for confounders (age, gender, atopy, smoking).

Results The mean inhalable dust concentrations modelled were $3.29 \mathrm{mg} / \mathrm{m} 3$ (GSD: 3.04), cumulative exposure in current job was $26.23 \mathrm{mg} / \mathrm{m} 3-y r$ (GSD: 2.94) and cumulative exposure over all jobs was $24.07 \mathrm{mg} / \mathrm{m} 3-\mathrm{yr}$ (GSD: 2.94). The mean age of predominately male workers was 38 years, among whom $76 \%$ were non-smokers and 50\% atopic. Work-related ocularnasal symptoms were associated, in a dose-dependent manner, with exposure groups (EGs) categorized according to current dust particulate levels (medium, 4.68-4.71 mg/m3: OR = 3.45, 95\% CI: 1.21 - 10.63; high, >4.71 mg/m3: OR = 3.66, 95\% CI: 1.19 - 11.26) versus low EG ( $<4.68 \mathrm{mg} /$ $\mathrm{m} 3)$ in adjusted models. Work-related asthma symptoms were associated with mean cumulative exposure $(\mathrm{OR}=1.01,95 \%$ CI: $1.00-1.02)$ in adjusted models. FeNO was associated with cumulative exposure in current job (quartile 2, OR= 1.23, 95\% CI: $1.02-1.51$; quartile $4, \mathrm{OR}=1.23,95 \% \mathrm{CI}$ : $1.02-1.49$ ) as well cumulative exposure across all jobs in the factory (quartile $4, \mathrm{OR}=1.22,95 \% \mathrm{CI}: 1.01-1.48$ ) versus quartile 1 in adjusted models.

Conclusion This study demonstrated positive exposure-response relationships between wood dust particulate for both workrelated ocular-nasal symptoms as well as asthma, suggestive of an acute irritant.

\section{P-180 COHORT PROFILE: THE DANISH OCCUPATIONAL MEDICINE COHORT - LONG-TERM PROGNOSIS FOR PATIENTS WITH WORK-RELATED DISORDERS}

${ }^{1}$ Vita Ligaya Dalgaard, Morten Vejs Willert, Marianne Kyndi, Jesper Medom Vestergaard, Johan Hviid Andersen, David Høyrup Christiansen. 'Aarhus BSS, Aarhus University Council, Denmark

\subsection{6/OEM-2021-EPI.222}

Objective Danish departments of occupational medicine currently receive about 9,000 patients each year referred due to suspected work-related disorders. In Denmark and internationally there is a lack of follow-up studies investigating the longterm prognosis of employees with work-related disorders. The purpose of this cohort is to facilitate long-term prognostic studies.

Methods The cohort was created through The Danish National Patient Register and comprises all patients seen in Danish Departments/units of Occupational Medicine from 2000-2018 $(\mathrm{N}=145,390)$. Numerous register data are included in the database from five years prior to time of inclusion until 2018. Examples of register data are; information on work, sickness absence and disability as well as number and type of contacts to health services, hospital admissions, income, education, type of occupation, social status, death and cause of death. Job Exposure Matrices (JEMs) on physical and psychosocial work exposure, life styles and other matrices are also available. The cohort will be updated with new patients regularly.

Results Currently the cohort comprises seven major groups: musculoskeletal $(\mathrm{n}=51.056)$, mental health $(\mathrm{n}=28.212)$, lung $(\mathrm{N}=12.274)$, skin $(\mathrm{N}=6.544)$, nervous system $(\mathrm{N}=5.513)$, cancer $(\mathrm{N}=1.566)$, and others $(\mathrm{n}=40.225)$. Preliminary analyses across groups suggest that the labor market attachment has been high among the majority (about 75\%) of patients during the years prior to the year of inclusion. However, results indicate that the main diagnostic groups, with the exception of skin patients, do not return to comparable levels of labor market attachment during the years following referral and diagnosis.

Conclusions The Danish Occupational Medicine Cohort is an open source dataset available to researchers interested in longterm follow-up on patients with work-related disorders. Preliminary analyses indicate most diagnostic groups do not return to prior levels of labor market attachment during the years following referral and diagnosis from a department of occupational medicine. 